\title{
Generalized Structural Scheme of the Measurement Converter Using an Iteratively Integrating Conversion Method
}

\author{
Igor Sergeyev \\ Aviation Computer-Integrated Complexes Department \\ National Aviation University \\ Kiev, Ukraine \\ sergeyevi@i.ua
}

\begin{abstract}
Generalized structural scheme of the measurement converter which uses iteratively integrating conversion method is described. Analysis of its conversion equation and errors was produced. Basic expressions for the calculation are listed. It is shown that the conversion equation for the steady state does not depend on the conversion coefficients of direct circuit blocks, which affect only a dynamic quality of converter that allows you to set low requirements to the said block at high precision converter as a whole.
\end{abstract}

Keywords-generalized structural scheme; iteratively integrating conversion method; conversion equation; integrator; iterative additive correction of errors; dynamics of the iterative process; steady state

\section{INTRODUCTION}

An important task for many automation systems is a navigation and motive control.

As known, motion control, which is also referred to as "servo control" or "robotics" is used in industrial processes to move a specific load in a controlled fashion. These systems can use five main types of actuators - hydraulic, pneumatic, electrical, thermal or magnetic and mechanical. An actuator is a type of motor that is responsible for moving or controlling a mechanism or system.

It is operated by a source of energy, typically electric current, hydraulic fluid pressure, or pneumatic pressure, and converts that energy into motion. The choice of the actuator type (i.e. the device that provides the energy to move the load) is based on power, speed, precision, and cost requirements. Electromechanical systems are typically used in high precision, low to medium power, and high-speed applications. These systems are flexible, efficient, and cost-effective. Motors are the actuators used in electromechanical systems. Through the interaction of electromagnetic fields, they generate power. These motors provide either rotary or linear motion.

This type of system is a feedback system, which is used to control position, velocity, and/or acceleration. The controller contains the algorithms to close the desired loop (typically position or velocity) and also handle machine interfacing with inputs/outputs, terminals, etc. The drive or amplifier closes the inner loop(s) (typically velocity or current) and represents the electrical power converter that drives the motor according to the controller reference signals. The motor can be rotary or linear. The motor is the actual electromagnetic actuator, which generates the forces required to move the load. Feedback elements such as tachometers, encoders and resolvers, are mounted on the motor and/or load in order to close the various servo loops.

\section{Problem Statement}

Among the many devices in systems of navigation and motive control, an important role is played by the various measurement converters. Reduction of level of the errors of measuring converters are very important for improving the metrological parameters of such systems.

Very often high conversion accuracy of measuring converters is provided by using special materials, application of special technology of its production or special design of converter elements performance [1], but this not always allows achieving the needed linearity of transfer function with sufficient accuracy.

Among the many methods of increasing the accuracy of measuring converters structural methods are very important [1].

Structural methods of improving the accuracy include use of circuits in which it is possible to eliminate or reduce the influence of some of the errors most unstable blocks and elements for the total error of the device.

A significant place among structural methods to increase the accuracy of measuring converters occupy iterative methods of error correction based on a well-developed in mathematics the theory of iterative methods.

At the same time, the measurement technique is very widely used various integrating converters, due to their advantages such as high accuracy, noise immunity and sensitivity, simplicity, reliability, low cost, etc. 
Combining methods of integrating conversion and additive iterative correction of errors has generated a method called by the author [2], [3] iteratively integrating conversion method and devices using this method - iteratively integrating converters (IIC). Many of iteratively integrating converters [4] possess high metrological parameters.

An analysis of the generalized structural scheme to improve the parameters of the device, which are developed.

\section{Solution of the Problem}

Simplified generalized structural scheme of iteratively integrating converter shown in Fig. 1, which was offered by the author in work [2]. Here in the simplest case $X$ is the input value; $Y$ is the output value; $\mathrm{OC}$ is output converter, $\mathrm{BC}$ is back converter; $\mathrm{K}_{\mathrm{BC}}$ is transfer coefficient of $\mathrm{BC}$; $\mathrm{SW} 1$ and SW2 are switches, $\mathrm{SH}$ is sample-and-hold device; $T 1$ is time interval of integration of the input variable $X ; T 2$ is time interval of integration of the value $\beta Y$. The values of $T 1$ and $T 2$, along with $X$ are input values.

The work comes in cycles. Each cycle is a step of iteration. It consist of the integration time $T 1$ by integrator I of variable $X$ through a non-inverting input, integration time $T 2$ by integrator I of variable $\mathrm{K}_{\mathrm{BC}} Y$ through an inverting input and memorizing by the sample-and-hold device $\mathrm{SH}$ of the integrator output value upon receipt at the end of the cycle of strobe $T 3$. This value enters through the output converter OC to output of converter during the subsequent conversion cycle.

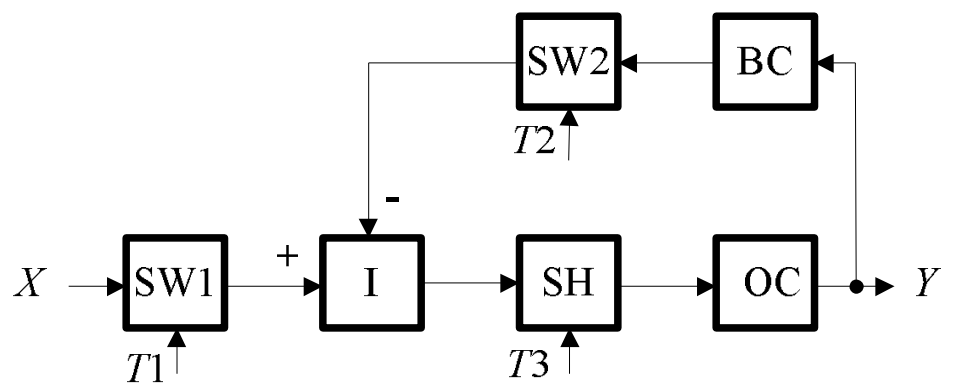

Fig. 1. Simplified generalized structural scheme of iteratively integrating converter.

Without dwelling on the details of the dynamics of the iterative process of establishing an output value that were discussed in detail in [2] - [5], we indicate only that after a few cycles of the transition process ends and the converter output value is given by

$$
Y=\frac{T 1}{T 2 \beta} X
$$

From this expression, which is an equation of the conversion of the simplified generalized structural scheme of iteratively integrating converter, it follows that the steady-state output value does not depend on the transformation coefficients of direct circuit blocks I, $\mathrm{SH}$ and $\mathrm{OC}\left(\mathrm{K}_{\mathrm{I}}, \mathrm{K}_{\mathrm{SH}}\right.$ and $\mathrm{K}_{\mathrm{OC}}$ respectively).

Another variant of the simplified generalized structural scheme is shown in work [6].

In general, the input and output values of IIC, and input and output values of the integrator, the sample-and-hold device and output converter may be of various nature. Thus, for example, integrable values may be [7] the electric current, voltage, the oscillation frequency, the angle of the shaft rotation, the angular or linear displacement, velocity or acceleration of the displacement, force or moment of forces, the stream of solid, liquid or gaseous substance, electrical energy, radiation flux, etc. The outputs of the integrators can also be very diverse. For electrical integrators the output signal can be voltage, current, or charge; for pneumatic integrator with an input value given by the air flow or gas, the output value is the volume of gas or pressure in the accumulator, and if the input signal is in the form of pressure pulses the output value is the counter readings; output of electronic or electromechanical integrators with different input signals can be counter readings, current or voltage; mechanical integrators to the input source in the form of a solid or liquid flow substances are output volume or weight of incoming substances; mechanical integrators with elastic elements are output displacement proportional to the integral of the rate of deformation of the elastic element; in electrochemical integrators the output value is a change of state of the electrodes by passing electric current through them, in the formation of a solid, liquid or gaseous phase in areas near of electrodes due to the electrochemical reactions, thus the total mass of the extracted substances is proportional to the quantity of electricity passing through the integrator, etc. etc.

As a result of the above, IIC in the general case should have additional units performing the intermediate conversion of physical values, as well as large-scale converters of various physical values. More complex generalized structural scheme of IIC proposed in work [4] and containing these blocks, is shown in Fig. 2.

The input values are $X, Z 1$ and $Z 2$, and the output value $Y$. The circuit consists of two parts. The first part contains the blocks that perform basic functions: integrator I, sample-andhold device SH, switches SW1 and SW2 switches. The second part contains auxiliary converters $-\mathrm{CV}_{\mathrm{X}}, \mathrm{CV}_{\mathrm{Z} 1}, \mathrm{CV}_{\mathrm{Z} 2}, \mathrm{CI}_{1}$, $\mathrm{CI}_{2}, \mathrm{CO}_{1}, \mathrm{CO}_{2}, . ., \mathrm{CO}_{m}, \mathrm{BC}$, inverter INV, adder ADD, having transfer coefficients, respectively, $\mathrm{K}_{X}, \mathrm{~K}_{Z 1}, \mathrm{~K}_{Z 2}, \mathrm{~K}_{\mathrm{Cl1}}, \mathrm{K}_{\mathrm{Cl} 2}$, $\mathrm{K}_{\mathrm{CO} 1}, \mathrm{~K}_{\mathrm{CO} 2}, . ., \mathrm{K}_{\mathrm{COm}}, \beta, \mathrm{K}_{\mathrm{INV}}, \mathrm{K}_{\mathrm{ADD}}$. 
The work of the converter is carried out cyclically. In each cycle the integration is performed of the input variable $X$, which is coming to an integrator I through blocks $\mathrm{CV}_{X}, \mathrm{SW} 1$, $\mathrm{CI}_{1}, \mathrm{INV}, \mathrm{ADD}$ and $\mathrm{CI}_{2}$ for a time $T 1$, a predetermined by input value $Z 1$; the integration is performed of the input variable $Y$, which is coming to an integrator I through blocks $\mathrm{BC}, \mathrm{SW} 2, \mathrm{CI}_{1}, \mathrm{INV}, \mathrm{ADD}$ and $\mathrm{CI}_{2}$ for a time $T 2$, a predetermined by input value $Z_{2}$, or the integration is performed of the input variable $Y$, which is coming to an integrator I through block $\mathrm{CV}_{Z 2}$ for a time $T 2$, a predetermined by output value $Y$ (in this case, the output value of the back converter $\mathrm{BC}$ is interval time); and sampling (for short time interval T3) the output value of integrator I by sample-andhold device $\mathrm{SH}$. The resulting output value $Y$ is unchanged for a subsequent cycle of operation.

Let us analyze the generalized scheme, considering all the blocks of an ideal, that is, for the case where the transfer coefficients of all the blocks are constant. This makes it possible to analyze the operation of the converter depending on the input values. values:

The following cases are combinations of input and output

- $\quad X, Z 1, Z 2$, and $Y$ are constant values;

- $X=X(t)$ is variable value; $Z 1, Z 2$ and $Y$ are constant values;

- $\quad X, Z 1$ and $Z 2$ are constant values, $Y=Y(t)$ is variable value;

- $\quad X=X(t)$ and $Y=Y(t)$ are variable values, $Z 1$ and $Z 2$ are constant values.

Let us analyze the case where the $X, Z 1, Z 2$ and $Y$ are constant values. (Analysis of the converter for other cases combinations of input and output values as well as the analysis taking into account the imperfection of all its blocks will be devoted the following publications of the author).

Let us assume the output value $Y=Y_{0}$. Let one of the input values change abruptly. For the $n$-th cycle after such a jump can write an expression for determining an output value in the form of

$$
Y_{n}=X K_{X} Z 1 K_{Z 1} \prod_{i=1}^{m} K_{i}+Y_{n-1} Q
$$

where $Q=1-Z 2 K_{Z 2} \beta \prod_{i=1}^{m} K_{i} ; m$ is covered by the feedback number of direct chain blocks $\mathrm{CO}_{1}, \mathrm{CO}_{2}, \ldots, \mathrm{CO}_{m} ; i$ is block number of direct chain blocks; $i=\overline{1, m}$.

Expression (1) represents an inhomogeneous linear differential equation of the first order, which, as known, it can be solved, for example, using the $Z$-transform. A result we get

$$
Y_{n}=X K_{X} Z 1 K_{Z 1} \prod_{i=1}^{m} K_{i} \sum_{j=1}^{n} Q^{j-1}+Y_{0} Q^{n}
$$

where $j$ is the serial number of the conversion cycle (after considered a jump of the input variable), $j=\overline{1, n}$.

Another, more obvious, by solving the equation (2) is the following. Equation (1) can be used as a recursion formula, for which a number (2) is constructed directly.

The strictness such solutions is proved by the method of mathematical induction. Because simplicity is the proof here will not be considered.

Expression (2) represents a sum of a geometric progression which converges on condition $|Q|<1$, and decreases at performance of the same conditions, a member $Y_{0} Q^{n}$.

Using known for a geometric progression formulas and recurrence formula (2), we write the expression for the output value of the converter at the end of the first conversion cycle after considered a jump of the input variable in the form of

$$
Y_{1}=X K_{X} Z 1 K_{Z 1} \prod_{i=1}^{m} K_{i}+Y_{0} Q
$$

at the end of the second conversion cycle

$$
Y_{1}=X K_{X} Z 1 K_{Z 1} \prod_{i=1}^{m} K_{i}(1+Q)+Y_{0} Q^{2},
$$

at the end of the $n$th conversion cycle

$$
\begin{aligned}
Y_{1}=X K_{X} Z 1 K_{Z 1} \prod_{i=1}^{m} K_{i} \sum_{j=1}^{n} Q^{j-1}+Y_{0} Q^{n} \\
=\frac{X K_{X} Z 1 K_{Z 1}}{Z 2 K_{Z 2} \beta}\left(1-Q^{n}\right)+Y_{0} Q^{n},
\end{aligned}
$$

in steady state $(n \rightarrow \infty)$

$$
Y_{\infty}=\lim _{n \rightarrow \infty} Y_{n}=\frac{X K_{X} Z 1 K_{Z 1}}{Z 2 K_{Z 2} \beta} .
$$

This expression represents the conversion equation of converter in static (in steady state), and the expression (2),..., (5) reflect the dynamics of the transitional process of establishing the output value after a sudden change of input value.

From equations (2) and (6) we can write the expression for determining the relative error caused by the finite conversion time $(n \neq \infty)$, in the form of

$$
\gamma_{n}=\frac{Y_{n}-Y_{\infty}}{Y_{\infty}}=-\frac{\Delta Y Q^{n}}{Y_{\infty}}
$$

where $\Delta Y=Y \infty-Y_{0}$, as well as an expression to determine the number of cycles at which this error becomes less than the specified: 


$$
n=\left[\frac{\ln \left|\frac{\gamma_{n} U_{\infty}}{\Delta U}\right|}{\ln |Q|}\right]+1 .
$$

The square brackets indicate that we take the integer part of the value of the expression in these brackets.

It follows from the above considerations that the speed of the transient process higher, if the $Q$ value is closer to zero. The condition $Q=0$ is the condition for the maximum speed the transitional process, which in this case ends in one cycle.

From these equations, which describe the formation of the output value of the iterative converter, and consideration of converter operation in each cycle can be stated as follows.

In the device is carried the conversion with the iterative additive correction of conversion error [1], in which as a test signal using the output value of the device. The error correction in the converter is iterative, because the output value step by step through closer to a predetermined steadystate value. The error correction in the converter is also additive, because the correction is entered by summing, which is performed using an integrator.

\section{CONCLUSION}

From the analysis follows that as a result of an iterative additive error correction in an measurement converter which uses an iteratively integrating conversion method which was examined the conversion equation for the steady state does not depend on the conversion coefficients of direct circuit blocks $\left(\mathrm{K}_{\mathrm{Cl} 1}, \mathrm{~K}_{\mathrm{Cl} 2}, \mathrm{~K}_{\mathrm{CO} 1}, \mathrm{~K}_{\mathrm{CO} 2}, . ., \mathrm{K}_{\mathrm{COm}}, \mathrm{K}_{\mathrm{INV}}, \mathrm{K}_{\mathrm{ADD}}\right)$, which affect only a dynamic quality of converter that allows you to set low requirements to the said block at high precision converter as a whole.

\section{REFERENCES}

[1] Yu. Tuz, The Structural Methodsof Impruving Accuracy of Measuring Devices, manual, Kyiv. Vyshcha Shkola, 1976. (in Russian).

[2] Yu. Tuz and I. Sergeyev, "An iterative converter of time interval to the voltage", "Measurement Equipment", no. 7, pp. 15-17, 1976 (in Russian).

[3] I. Sergeyev, "Analysis of the ADC with a dynamic integrator", "Measurement Equipment", no 6, pp. 38 - 40, 1976 (in Russian).

[4] I. Sergeyev, Research and Development of Integrating Measurement Converters with the Iterative Additive Correction of Errors, Ph. D. (Engineering) Thesis, Kyiv Politechnical Institute, Kyiv, Ukraine, 1978 (in Russian).

[5] V. Gubar, I. Sergeyev, and V. Fediv, USSR Patent 661,525, G 06 G, 7/26, 05.05.79. bull. 17 (in Russian).

[6] V.S. Gutnikov and A.V. Klementiev, "Performance improvement integrating analog-to-digital converters, carried out along a closed structure. Measurement converters": Interuniversity collection of proceedings, vol. 1, Omsk Polytechnic Institute, 1975 (in Russian).

[7] V.M. Plotnikov, V.A. Podreshetnikov, and L.N. Teteriatnikov, Integrating measuring instruments. Moscow, "Mashinostroenie", 1977, (in Russian) 